\title{
Motion Behavior and Range of Motion when Using Exoskeletons in Manual Assembly Tasks
}

\author{
Estela PEREZ LUQUE ${ }^{1, a}$, Dan HÖGBERG ${ }^{\mathrm{a}}$, Aitor IRIONDO PASCUAL ${ }^{\mathrm{a}}$, Dan \\ LÄMKULL $^{\mathrm{b}}$ and Francisco GARCIA RIVERA ${ }^{\mathrm{a}}$ \\ a School of Engineering Science, University of Skövde, Skövde, Sweden \\ ${ }^{\mathrm{b}}$ Global Strategy \& Process Development, Volvo Cars, Gothenburg, Sweden
}

\begin{abstract}
Although the automation level is high within the automotive industry, there are still a large number of manual tasks, especially is the final assembly of the vehicle. Overhead assembly operations is an example of a problematic manual task that can cause workers to develop musculoskeletal disorders in the shoulder complex. Exoskeletons may be a solution to reduce the risk for developing musculoskeletal disorders from the work tasks. This study evaluates and compares how the use of three different passive upper body exoskeletons affects the range of motion (ROM) of workers at overhead assembly tasks. An experiment consisting of three tasks was set up in order to analyze the differences between the models. Seventeen subjects were involved in the study. Interviews, observations, videos and motion capture recordings were the methods of collecting data. The results show agreement from all the subjects that the exoskeletons help the worker at this specific assembly operation. The results also show that different exoskeleton models cause different levels of ROM reductions. The subjects' opinions about how the different exoskeletons influence the ROM corresponds with the analysis of the motion capture data. Positive and negative aspects of each exoskeleton from a ROM and an implementation point of view are discussed. In general, the results indicate that the exoskeleton models can be applicable for the type of work tasks studied. However, the exoskeletons would benefit from further development in order to decrease ROM limitations and therefore cover a larger number of different manual assembly tasks.
\end{abstract}

Keywords. Exoskeleton, range of motion, overhead operation, musculoskeletal disorders, ergonomics.

\section{Introduction}

According to the Sixth European Working Conditions Survey (EWCS), which measured, analyzed and identified the working conditions across 35 European countries in 2015, posture-related problems are the most frequent risk within the physical environment category [1]. In the survey, the exposure to different physical risks in the work area is divided into three categories: posture-related, biological and chemical, and environmental (e.g. vibrations, noise, and high and low temperatures). The posturerelated category, which covers exposure to vibrations, tiring positions, lifting people, carrying heavy loads and repetitive movements, is the most prevalent risk. In particular, within posture-related risks, repetitive movement of hands and arms is the most reported

1 Estela Perez Luque. estela.perez.luque@his.se 
issue $(61 \%)$ from workers (men and women). The EWCS survey highlights the need to address and prevent the risks for work-related musculoskeletal disorders (WMSDs) in industry.

Overhead work is defined as any work that requires the hands in a position above the height of the shoulders, in essence, above the head [2]. Postures with greater angle than $60^{\circ}$ of shoulder flexion or abduction, although the hands may not be over the acromial height, are defined as awkward since this type of operation constitutes a challenge on the shoulder complex [2]. These postures are not only awkward, but they also include limitations in the range of motion (ROM), and might lead to disorders from performing repetitive tasks. Several studies have demonstrated the evidence of the association between these exposures and the development of pathologies as tendonitis, impingement, rotator cuff syndrome or muscle fatigue, which can lead to the inability to work in short or long term [3].

According to the Bureau Labor Statistics, shoulder and low back musculoskeletal disorders caused an average of twelve lost working days per affected person, between 2014-2015 [4]. All in all, overhead operations in the industry can cause musculoskeletal disorders, leading to human suffering and sick-leave, and result in loss of productivity and cause immense economic costs [5].

The use of exoskeletons in the industry may have the potential to reduce risks for WMSDs, for example in manual tasks that require overhead work. Exoskeletons to support manual work is a new technology with limited implementation in industrial practice, it is rather in a testing or experimental stage [6], [7]. So far, the main application of exoskeletons has been in the medical/rehabilitation field and in the military area [8]. However, the interest on exoskeletons in industry has increased due to its potential to support and enhance the capabilities of workers and reduce risks for WMSDs [9], [10]. Exoskeletons can be divided into different categories, depending on aspects such as: intended use, power requirements, form factors, or construction materials [11], [12].

Although numerous exoskeletons for industrial applications are available on the market, the investigations about their use have not evaluated all their possible effects so far [12]. Several studies focus on examining the muscle activity changes of muscle groups that are intended to be supported by the exoskeletons [7], [12]-[16]. The results indicate that exoskeletons do reduce the load levels on the supported muscles. However, there is a lack of research on other effects of using exoskeletons in industry, e.g. biomechanical load shifts to other muscles, longitudinal effects, postural changes, or limitations in the range of motion [17]-[21]. Range of motion (ROM) is defined as the available amount of movement, linear or angular, of a specific joint [22].

The present study assesses how the use of three different passive upper body exoskeletons affect the ROM of workers.

\section{Method}

\subsection{Approach}

An experimental study was designed to assess the possible effects on the ROM of workers while wearing an exoskeleton during manual assembly operations. The experiment included three tasks to perform with three different passive exoskeletons, and without wearing an exoskeleton. A physical testbed, located in an industry-like environment, was created to simulate working at a workplace (Figure 1). Kinematic data 
and subjective opinions of the subjects during the performance of the test tasks were measured to carry out the comparative analyses.

\subsection{Scenario}

The scenario of the experiment was to replicate a real workplace of overhead assembly operations in the automotive industry. The actual operation in the factory consists of attaching plastic plates on the underbody of the car. To replicate the real workstation and its space conditions, the testbed was designed with $164 \mathrm{~cm} \times 246 \mathrm{~cm} \times 200 \mathrm{~cm}$ (width $\mathrm{x}$ depth $\mathrm{x}$ height) dimensions (Figure 1). The subjects were standing below the upper plate to access the "underbody of the car" where four nuts were mounted in order to execute the screw assembly tasks (Figure 2). A mechanism enabled height adjustment of the upper plate when required.

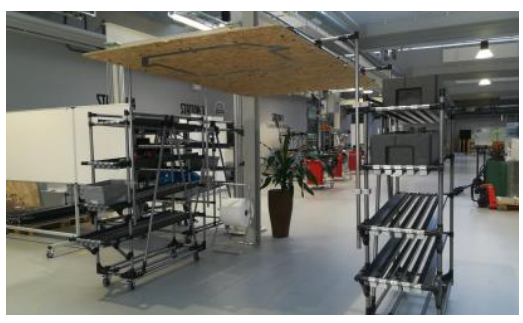

Figure 1. Testbed.

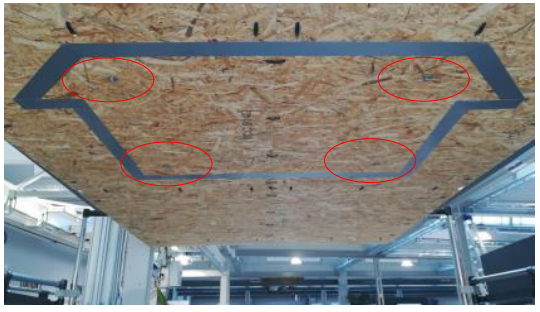

Figure 2. Nuts in upper plate for screw operations.

\subsection{Subjects}

17 healthy subjects (11 men and 6 women), with no recent reported injuries in the back and upper body, were involved in the experiment. Among the 17 subjects, 8 were operators from an automotive factory, 7 students from an upper secondary school, and 2 university students: mean age 25 (range 18-46) years and mean stature 174 (range 166190) $\mathrm{cm}$. All subjects provided informed consent for the performance of the experiment, and they were not familiar with the tasks and equipment involved in the experiment.

\subsection{Task descriptions}

The subjects performed three tasks, devised to assess the impact on the ROM when using exoskeletons, as well as the exoskeletons' versatility. The first and second task simulated overhead assembly work in a real factory, and the third task focused on the collection of ROM data.

Task 1. Rotation and overhead work. Task 1 consisted of doing a rotation movement for taking the tool and fasteners and then perform the screw operations. The subject stands under the upper plate and turns his/her back to the side of the testbed where the tool is, keeping the legs in a static position for this first movement of rotation (Figure 3a). After taking the tools, the subject performs the screw operations inserting the screws into the nuts placed in the upper plate.

Task 2. Bending and overhead work. Task 2 is similar to Task 1, with the difference of taking the tool from the floor. In this case, the subject has to perform a bending movement, flex the back and legs in order to reach the tool (Figure 3b). 
Task 3. ROM. The subject first stretches arms up and down and then performs circular movements with the arms (Figure 3c).
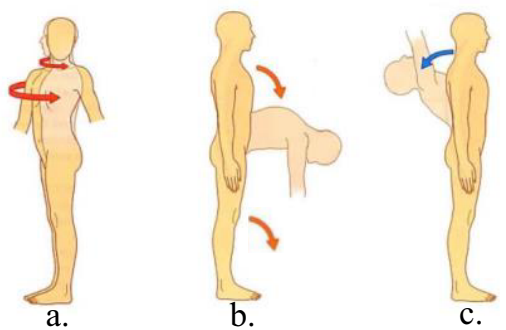

Figure 3. Involved movements in the tasks (a. Rotation; b. Bending; c. Stretching).

Task 1 and Task 2, based on real workplace observations, aim to simulate overhead assembly work at the factory while considering possible difficulties such as the need to be able to pick up items from the floor. Task 3 aims to observe and measure ROM of the subjects when making more extreme or unusual movements. In addition to the task descriptions, the subjects received no specific instructions for the task execution. This in order to keep the performance as close as possible to a real assembly situation.

\subsection{Apparatus and instrumentation}

\subsubsection{Exoskeletons}

Three different models of upper body exoskeletons were used in the experiment (Figure 4). The exoskeletons were passive, meaning that no external power supply was required, and they were developed to provide help to workers and reduce the effort during daily tasks. Even though the three exoskeletons were different in design, their core functionality was equal: giving lift assistance for upper arm flexion, based on mechanical components like springs and lever mechanisms. They were worn similar to a backpack, close to the body with attachments in the arms, lower back and chest. They offered force support to raise the arms and to keep them raised. In this way they aimed to reduce the effort in the shoulder and neck region. Figure 4 shows the three exoskeleton models, and Table 1 describes the main characteristics of each model.

Table 1. Characteristics of the exoskeleton models.

\begin{tabular}{cccc}
\hline Model & A & B & C \\
\hline Weight & $\approx 4.3 \mathrm{~kg}$ & $\approx 2 \mathrm{~kg}$ & $\approx 4 \mathrm{~kg}$ \\
Lift Assistance & 4 assistance levels & Adjustable assistance & 7 assistance levels \\
Height Range & S-M-L sizes, $152-193 \mathrm{~cm}$ & $160-190 \mathrm{~cm}$ & S/M and L/XL sizes \\
Examples of & Ford Motor Company, & Volkswagen & \\
implementation & BMW & & \\
\hline
\end{tabular}



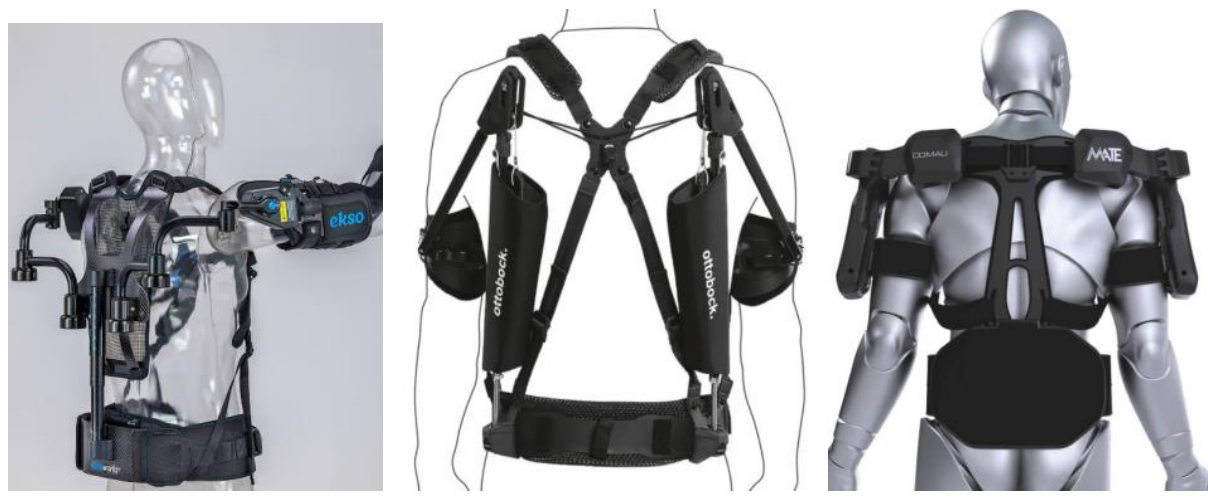

Figure 4. The exoskeleton models. From left to right, Model A [23]; Model B [24]; Model C [25].

\subsubsection{Instrumentation: Motion capture equipment and tools}

Xsens MVN Awinda, which is a portable inertial measurement unit (IMU) based motion capture system was used to measure kinematic data [26]. The MVN system is able to measure and record kinematic data including body segment positions, joint angles, and center of mass [27], [28]. The motion sensors were mounted on the subjects using flexible straps, which meant that the motion capture equipment was adapted to the anthropometry of each subject. The motion capture equipment was believed to have no influence on the subject's motion behavior or ROM while performing the tasks.

An electric screwdriver (approx. $2 \mathrm{~kg}$ ) was used to mount the screws, similar to the tool used in the corresponding assembly operations in the real factory.

\subsection{Procedure}

To get started, the experiment procedure was briefed to each subject.

The first step for each subject was the setup of the motion capture system. Segment calibration was performed to align the segments of the subject to the motion trackers. A good calibration performance was essential to ensure accurate results [26].

Next, the subject executed the three tasks four times. The first performance of the tasks was done without using any exoskeleton, just wearing the sensors of the motion capture system. In this way the subject got familiar with the tasks and the tools. After that, the performance of the three tasks was carried out again with each one of the exoskeletons: Model A, Model B, and Model C, respectively. To summarize, each subject performed the three tasks four times: first just wearing the motion capture system, followed by wearing Model A, Model B, and last Model C.

Each of the exoskeletons was adjusted to suit the subject's bodily characteristics. The subjects started the tests in a standing position under the upper plate without the electric screwdriver tool. At the beginning of the first and the second task, the subjects reached the tool and screws with different movements (taking the tool from the side or from the floor) and then they went on with the operations.

After conducting the three assembly tasks four times, the last step consisted of interviewing the subjects, in order to gather a complete view of the subject's opinions about each exoskeleton and their differences. However, after performing each batch of assembly tasks, the subjects were also asked about their impressions when using each of the models. It was instructed to the subjects to execute the tasks naturally within the 
provided indications. All subjects were not only recorded with the motion capture system but also with a video camera. The duration to perform the whole experiment, including the motion capture system set up and changes of exoskeletons, was around 50-60 min per subject.

\subsection{Data collection}

Qualitative and quantitative data were collected and analyzed to assess how the exoskeletons influenced the ROM of the subjects.

The qualitative data consisted of the observations (general but not accurate insight of the human behavior) and mainly of the interviews done after performing the experiments. These semi-structured interviews were carried out once the subjects were done with all the tasks. The questions were related to performing the specific screwing task, to the limitations on movements when taking the tool from the side or floor and during the task, main advantages and disadvantages, and force assistance provided by each model. The quantitative data was collected by the motion capture system.

\subsection{Data analysis}

The quantitative data analysis consisted of analyzing specific data from the motion capture records. The joint angles of both right and left shoulders were studied since they are the main affected areas at overhead assembly operations. The motion capture records provided data of three different shoulder joint movements: flexion/extension, abduction/adduction, and internal rotation/external rotation (Figure 5). A statistical analysis, based on percentiles, was done to compare and observe significant changes in the subjects' ROM when using the different exoskeletons models during the performance of the tasks. Percentile studies are used to analyze a large number of observations and continuous probability distributions [29]. Examples of other studies that follow a similar approach for motion data analysis and presentation are: [30]-[32]. A percentile is a measure that indicates the value below which a given percentage of observations in a group of observations falls [29]. With this method, it is possible to observe the distribution of the data points (right and left shoulder joint angles) of each subject, with each of the exoskeleton models and without, and to analyze possible changes with each exoskeleton model in the same tasks, as well as to see what the movement trends are. For example, if by wearing any of the models, the exoskeleton keeps the arms raised up longer times due to the lift assistance support, or if the movement and trend are similar to the "optimal" or natural performance (without wearing an exoskeleton). For Tasks 1 and 2, flexion/extension is the movement studied, and in Task 3 abduction/adduction. The choice of what body movements to analyze was based on the main movements involved in each of the three tasks. 

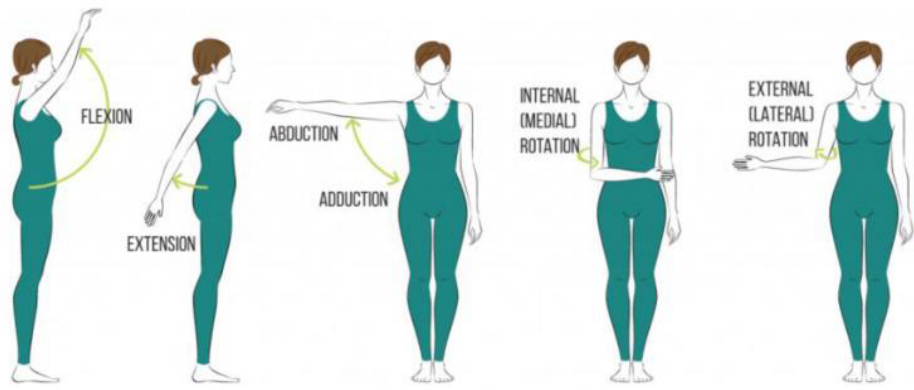

Figure 5. Shoulder joint movements [33].

\section{Results}

\subsection{Qualitative results}

Table 2 summarizes the subjects' preferences per exoskeleton model, divided into: Best option, Second option, and Third option. The preference order was based on each subject's experienced comfort with each model, limitations in ROM, and lift assistance (force support) during the experiment.

Table 2. Exoskeleton model preferences.

\begin{tabular}{cccc}
\hline Preferences & Model A & Model B & Model C \\
\hline Best option & 9 & 12 & 0 \\
Second option & 4 & 3 & 6 \\
Third option & 4 & 2 & 11 \\
\hline
\end{tabular}

Model B was the favorite for the subjects regarding ROM (12 subjects), followed by Model A (9 subjects) and Model C (which no subject selected as best option). Four of the subjects chose both Model A and B as the best option.

Table 3 highlights the most repeated positive and negative aspects of each exoskeleton model by the subjects.

Table 3. Positive and negative points: Model A, Model B and Model C.

\begin{tabular}{|c|c|c|}
\hline Exoskeleton Model & Positive points & Negative points \\
\hline Model A & $\begin{array}{l}\text { Lift assistance } \\
\text { Comfort } \\
\text { Adjustment to the arms }\end{array}$ & $\begin{array}{l}\text { Null flexibility in the back } \\
\text { Back, shoulders and sides discomfort } \\
\text { Heavy } \\
\text { Clumsy feeling }\end{array}$ \\
\hline Model B & $\begin{array}{l}\text { Comfort } \\
\text { Full range of motion } \\
\text { Lightweight }\end{array}$ & $\begin{array}{l}\text { Adjustment to the arms } \\
\text { Shoulder discomfort }\end{array}$ \\
\hline Model C & $\begin{array}{l}\text { Earlier lift assistance } \\
\text { Adjustment to the arms and lower back }\end{array}$ & $\begin{array}{l}\text { Limitations in the range of motion } \\
\text { Back and shoulder discomfort } \\
\text { Limited support at overhead position } \\
\text { Clumsy and stuck feeling } \\
\text { Heavy }\end{array}$ \\
\hline
\end{tabular}

Model A has good lift assistance and adjustment to the arms. However, it also produced back, shoulder and side discomfort. Heavy and clumsy were other of the most repeated comments. Model B was the favorite one for most of the subjects. A textile material composition makes it the most lightweight of the three exoskeleton models. 
Subjects expressed that it was the model performing in a better way the natural movement and fitting on the body. However, the adjustment to the arms was considered a negative point by almost every subject. Model $\mathrm{C}$ was the exoskeleton that received most negative comments. Although the lift assistance started earlier, and the adjustment in the lower back and arms were qualified as the best of the three models, the design limits reduced ROM, and the performance movement got stuck, especially during Task 3. Moreover, Model $\mathrm{C}$ also produced discomfort in the back, and a clumsy feeling due to the weight.

Regarding the movements involved to reach the tool at the beginning of Tasks 1 and 2, no problems were obtained taking the tool from the side (Task 1). In contrast, reaching the screwdriver from the floor (Task 2) was not easy for all the subjects with the exoskeleton Model A and $\mathrm{C}$ due to experienced hip discomfort, a consequence of the lower back adjustments and the devices' weight. On the other hand, all subjects agreed that the use of these devices help at this specific overhead operation, although performing the experiment and tasks without any of the exoskeletons provided a full ROM.

\subsection{Quantitative results}

By doing a deeper analysis of the data collected by the motion capture equipment, it was possible to study the shoulder joint angles adopted by the subjects during the different tasks in the experiment. Results for Subject \#1 are shown in Figure 6-8. Only data for the first subject is presented due to space limitations. The results of the other subjects showed a similar pattern. The horizontal axis in the graphs describes the angular distribution in percentiles (P1, P5, P25, P50, P75, P95, and P99), and the vertical axis shows the shoulder joint angle. In Task 1 and Task 2 (Figure 6-7), the angle values go from -10 to 90 degrees, where this range corresponds to shoulder joint flexion/extension (Figure 5). In Task 3 (Figure 8), the angle values go from -20 to 180 degrees, where this range corresponds to shoulder joint abduction/adduction (Figure 5). Series 1 (green) corresponds to the first recordings of the experiment just wearing the motion capture equipment without an exoskeleton ("Mocap" in Figure 6-8). This represents the movements the subject is likely to have in real assembly work, performing the tasks without wearing any exoskeleton system. This is considered the "optimal" or natural movement, and constitutes a reference of which to compare the rest of the data. Series 2 (blue), Series 3 (grey) and Series 4 (yellow) correspond to the recordings with the exoskeleton Model A, B, and C respectively (Figure 6-8).

Figure 6 (Task 1) and Figure 7 (Task 2) show higher angle values in most of the percentiles for the models $\mathrm{A}, \mathrm{B}$ and $\mathrm{C}$. In general, there seems to be a higher number of points at higher flexion angles. This change appears to be more significant with the exoskeleton Model C. Model A and B instead describe values more similar to the "optimal performance" (Series 1), i.e. without the exoskeleton. 

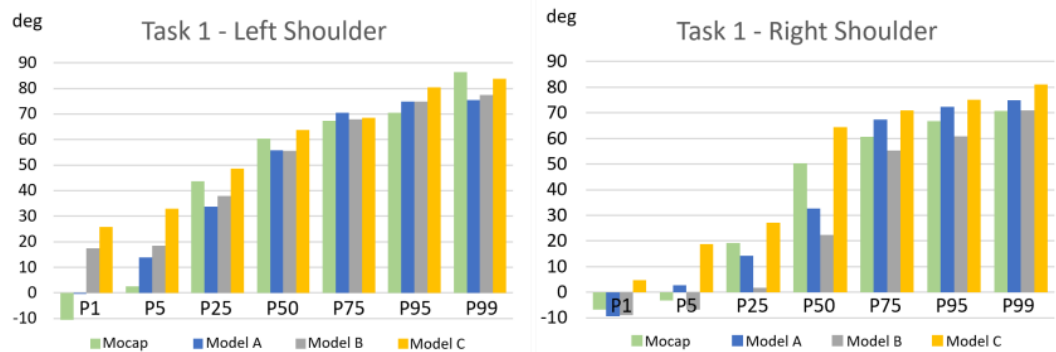

Figure 6. Left and right shoulder joint angle (flexion/extension) percentiles: Task 1, Subject \#1.
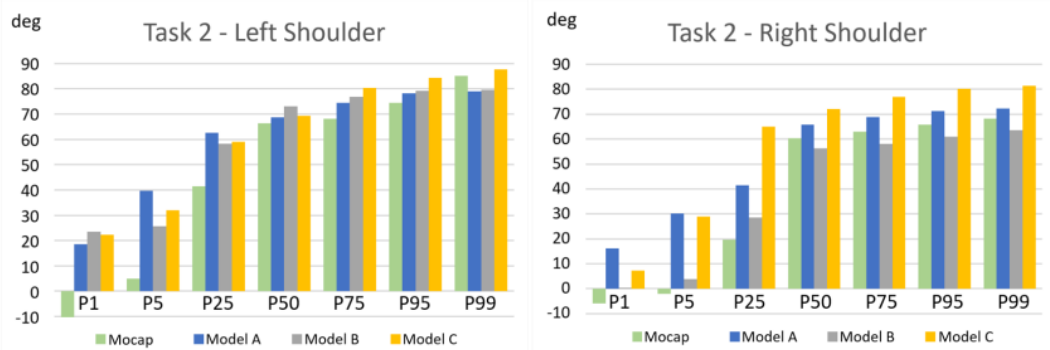

Figure 7. Left and right shoulder joint angle (flexion/extension) percentiles: Task 2, Subject \#1.
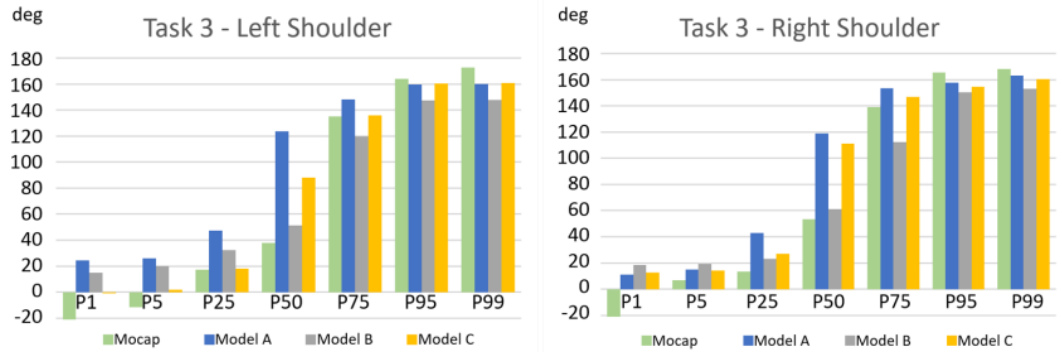

Figure 8. Left and right shoulder joint angle (abduction/adduction) percentiles: Task 3, Subject \#1.

During Task 3 (Figure 8), it can be observed higher angle values at the percentiles rank P25 to P75, which suggests that there might be a higher amount of medium values in the data sample, i.e. those corresponding to shoulder angles of abduction/adduction around $90^{\circ}$.

\section{Discussion}

\subsection{Task definition}

The experiment, the testbed and the tasks involved, were designed to replicate assembly operations and factory conditions in a realistic manner. The tasks were meant to simulate assembly operations that included body movements that enabled studying the range of motion while wearing exoskeletons system. Additional tasks could have been added in order to extend the study. This would, however, had even further increased the duration 
of the experiment, which was 50-60 minutes per person, which was considered an appropriate length when designing the study.

\subsection{Results}

Different exoskeleton models suited and affected the subjects differently. Both positive and negative aspects were registered when using each of the models and depending on the task. This corresponds with the results Baltrusch et al. obtained in their study [17]. None of the models received a completely positive assessment. According to the subjects' opinions, all of the models have some issues that need improvements.

The quantitative and qualitative results show that Model B is the exoskeleton with smaller changes in body motion behavior than Model $\mathrm{A}$ and $\mathrm{C}$, when compared to wearing no exoskeleton. Model B was subjectively also the preferred model from the ROM point of view, with more positive opinions than Model A and C. However, Model $\mathrm{B}$ has limitations regarding the adjustment to the subject's arms. When performing Task 3 , stretching the arms up and down and making circular movements, the adjustment of Model B slipped to the forearm, and therefore, the arms did not receive the lift assistance anymore. Although circular movements with arms are less likely movements in a real assembly operation, it is important to consider that the users of Model B are not able to stretch their arms up without causing ROM limitations. This problem can affect people with certain anthropometry, requiring them to raise their arms more than 90 degrees to do the overhead assembly operations. By improving this adjustment in Model B, the exoskeleton adjustment to the arms would not move to the forearm and the lift assistance would be applied on the correct body area all the time, i.e. the upper arm. Both Model A and $\mathrm{C}$ are heavier than Model $\mathrm{B}$, which was one of the main negative comments on those two exoskeletons. By reducing the weight of Model A and C, the exoskeletons would fit the users better, possibly reducing back, shoulder and side discomfort, as well as improving the overall performance of tasks requiring larger ROM (e.g. taking the tool from the floor).

To explain the changes on the movement trends shown in the percentile study, it can be said that for Task 1 and Task 2, where the joint values (degrees) increase from P75 to P99 for series 2, 3 and 4, the use of exoskeletons tends to keep both arms up due to the lift assistance. Similar to this, when performing the circular movements (Task 3), the subjects took more time doing an effort to overcome the lift assistance of the exoskeletons (around $90^{\circ}$ flexion/extension angle) to be able to move the arms back down.

According to the results, it can be said that the implementation of exoskeletons helps to support the arms at specific overhead assembly operations, although it might suppose changes to the natural performance (movements without exoskeleton). Nonetheless, when it comes to implementation in the industry, negative aspects of some of the models (Model A and C) should be considered. The clumsy and stuck feeling could cause psychological disorders if exoskeletons are used during long periods of time [34], [35]. In addition, the lower back, shoulder and side discomfort may suppose problems, and lead to other types of WMSDs as well as limitations in the worker's ROM. Consequently, implementing exoskeleton systems to reduce shoulder musculoskeletal disorders may lead to other types of discomfort or disorders, e.g. physical, as in the lower back region, or ROM limitations, and/or psychological negative effects. 


\subsection{Limitations}

The present study has a few limitations that should be indicated. The study compared three different exoskeletons in the context of only two tasks (overhead screwing operation and stretching), where only one of the tasks is a real industry example, during a short duration. These simulated tasks cannot involve aspects of the real working environments that could also influence the use of the exoskeletons, like the use of protective equipment, the pressure of takt times and/or the repetitiveness along periods of time. Regarding the results, although the experiment involved seventeen subjects, it is unknown if the same patterns of results would be obtained for more subjects. Additionally, there can be other promising upper body passive exoskeletons in the market or still in development [12], [36], with different designs to the ones that were assessed in this study, that may lead to better and/or different results.

\section{Acknowledgement}

This work has been made possible with the support from VINNOVA in the project VIVA - the Virtual Vehicle Assembler, and by the participating organizations. This support is gratefully acknowledged.

\section{References}

[1] A. Parent-Thirion et al., 6th European Working Conditions Survey: overview report, 2017 update. Luxembourg: Publications Office of the European Union, 2017.

[2] J. R. Grieve and C. R. Dickerson, Overhead work: Identification of evidence-based exposure guidelines, Occup. Ergon. 8, 2008, pp. 53-56.

[3] Musculoskeletal disorders and workplace factors, U.S. Department of Health and Human Services, Public Health Service, Centers for Disease Control and Prevention, National Institute for Occupational Safety and Health, Jul. 1997.

[4] Bureau of Labor Statistics, 2017, Number of Nonfatal Occupational Injuries and Illnesses Involving Days Away from Work by Selected Worker Case Characteristics and Musculoskeletal Disorder, Accessed: 17.11.2019. [Online]. Available: https://www.bls.gov/iif/soii-chart-data-2017.htm.

[5] M. A. Davis, Where the United States Spends its Spine Dollars: Expenditures on different ambulatory services for the management of back and neck conditions, Spine, vol. 37, no. 19, 2012, pp. 1693-1701.

[6] A. M. Dollar and H. Herr, Lower Extremity Exoskeletons and Active Orthoses: Challenges and State-ofthe-Art, IEEE Trans. Robot., vol. 24, no. 1, 2008, pp. 144-158.

[7] C. Wang, L. Ikuma, J. Hondzinski, and M. de Queiroz, Application of Assistive Wearable Robotics to Alleviate Construction Workforce Shortage: Challenges and Opportunities in Computing in Civil Engineering 2017, Seattle, Washington, 2017, pp. 358-365.

[8] B. S. Rupal, S. Rafique, A. Singla, E. Singla, M. Isaksson, and G. S. Virk, Lower-limb exoskeletons: Research trends and regulatory guidelines in medical and non-medical applications, Int. J. Adv. Robot. Syst., vol. 14, no. 6, 2017, p. 172988141774355.

[9] B. Marinov, What is an exoskeleton? Exoskeleton Report, Accessed: 15.10.2019. [Online]. Available: https://exoskeletonreport.com/what-is-an-exoskeleton/.

[10] J. Wesslèn, Exoskeleton Exploration: Research, development, and applicability of industrial exoskeletons in the automotive industry, Master Thesis of Production system area, School of Engineering, Jönköping University, Sweden, 2018.

[11] D. Kara, Industrial Exoskeletons: new systems, improved tech, increasing adoption, The Robot Report, Accessed: 01.11.2019. [Online]. Available: https://www.therobotreport.com/industrial-exoskeletons/.

[12] M. P. de Looze, T. Bosch, F. Krause, K. S. Stadler, and L. W. O'Sullivan, Exoskeletons for industrial application and their potential effects on physical work load, Ergonomics, vol. 59, no. 5, 2016, pp. 671681. 
[13] C.-J. Yang, J.-F. Zhang, Y. Chen, Y.-M. Dong, and Y. Zhang, A Review of exoskeleton-type systems and their key technologies, Proc. Inst. Mech. Eng. Part C J. Mech. Eng. Sci., vol. 222, no. 8, 2008, pp. 1599-1612.

[14] K. Huysamen, T. Bosch, M. de Looze, K. S. Stadler, E. Graf, and L. W. O'Sullivan, Evaluation of a passive exoskeleton for static upper limb activities, Appl. Ergon., vol. 70, 2018, pp. 148-155.

[15] E. Pirondini et al., Evaluation of the effects of the Arm Light Exoskeleton on movement execution and muscle activities: a pilot study on healthy subjects, J. NeuroEngineering Rehabil., vol. 13, no. 1, 2016.

[16] S. Alabdulkarim, S. Kim, and M. A. Nussbaum, Effects of exoskeleton design and precision requirements on physical demands and quality in a simulated overhead drilling task, Appl. Ergon., vol. 80, 2019, pp. $136-145$.

[17] S. J. Baltrusch, J. H. van Dieën, C. A. M. van Bennekom, and H. Houdijk, The effect of a passive trunk exoskeleton on functional performance in healthy individuals, Appl. Ergon., vol. 72, 2018, pp. 94-106.

[18] B. L. Ulrey and F. A. Fathallah, Subject-specific, whole-body models of the stooped posture with a personal weight transfer device, J. Electromyogr. Kinesiol., vol. 23, no. 1, 2013, pp. 206-215.

[19] B. L. Ulrey and F. A. Fathallah, Effect of a personal weight transfer device on muscle activities and joint flexions in the stooped posture, J. Electromyogr. Kinesiol., vol. 23, no. 1, 2013, pp. 195-205.

[20] N. Sylla, V. Bonnet, F. Colledani, and P. Fraisse, Ergonomic contribution of ABLE exoskeleton in automotive industry, Int. J. Ind. Ergon., vol. 44, no. 4, 2014, pp. 475-481.

[21] E. B. Weston, M. Alizadeh, G. G. Knapik, X. Wang, and W. S. Marras, Biomechanical evaluation of exoskeleton use on loading of the lumbar spine, Appl. Ergon., vol. 68, 2018, pp. 101-108.

[22] J. G. Konin and B. Jessee, Range of Motion and Flexibility, Physical Rehabilitation of the Injured Athlete, Elsevier, 2012, pp. 74-88.

[23] EksoVest - Upper Body Exoskeleton Supports Endurance, Ekso Bionics. Accessed: 21.10.2019. [Online]. Available: https://eksobionics.com/eksoworks/eksovest/.

[24] Paexo, Paexo. Accessed: 21.10.2019. [Online]. Available: https://www.ottobock.com/en/company/ottobock-industrials/paexo/.

[25] Comau, Comau. Accessed: 19.10.2019. [Online]. Available: https://www.comau.com/EN/MATE.

[26] Xsens MVN User Manual. Xsens, 2018.

[27] M. Schepers, M. Giuberti, and G. Bellusci, Xsens MVN: Consistent Tracking of Human Motion Using Inertial Sensing, p. 8.

[28] M. Paulich, M. Schepers, N. Rudigkeit, and G. Bellusci, Xsens MTw Awinda: Miniature Wireless Inertial-Magnetic Motion Tracker for Highly Accurate 3D Kinematic Applications, p. 9.

[29] R. J. Hyndman and Y. Fan, Sample Quantiles in Statistical Packages, Am. Stat., vol. 50, no. 4, 1996, pp. 361-365.

[30] I.-M. Rhén, M. Forsman, R. Örtengren, D. Högberg, and A. Keyvani, Ergonomic risk assessment in DHM tools employing motion data - exposure calculation and comparison to epidemiological reference data, Int. J. Human Factors Modelling and Simulation, vol. 6, 2018, p. 34.

[31] S. E. Mathiassen, J. Wahlström, and M. Forsman, Bias and imprecision in posture percentile variables estimated from short exposure samples, BMC Med. Res. Methodol., vol. 12, no. 1, 2012, p. 36.

[32] B. Juul-Kristensen, G.-Å. Hansson, N. Fallentin, J. H. Andersen, and C. Ekdahl, Assessment of work postures and movements using a video-based observation method and direct technical measurements, Appl. Ergon., vol. 32, no. 5, 2001, pp. 517-524.

[33] O. Kabel, Yoga for your body. Accessed: 11.10.2019. [Online]. Available: https://sequencewiz.org /2016/03/16/loosen-up-your-shoulders/

[34] Musculoskeletal disorders - EU-OSHA, European Agency for Safety and Health at Work. Accessed: 12.10.2019. [Online]. Available: https://osha.europa.eu/en/themes/musculoskeletal-disorders.

[35] V. Anderson et al., Musculoskeletal Disorders and Workplace Factors: A Critical Review of Epidemiologic Evidence for Work-Related Musculoskeletal Disorders of the Neck, Upper Extremity, and Low Back. 1997.

[36] A. Voilque, J. Masood, Jc. Fauroux, L. Sabourin, and O. Guezet, Industrial Exoskeleton Technology: Classification, Structural Analysis, and Structural Complexity Indicator, in 2019 Wearable Robotics Association Conference (WearRAcon), Scottsdale, AZ, USA, 2019, pp. 13-20. 